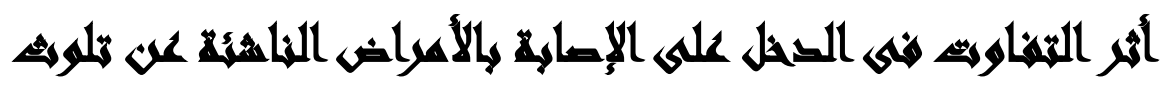 \\ المياه هنى هصر
}

[1人]

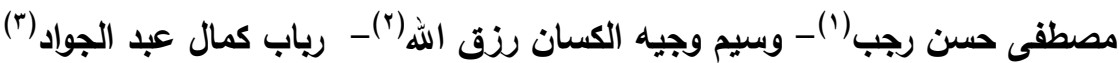

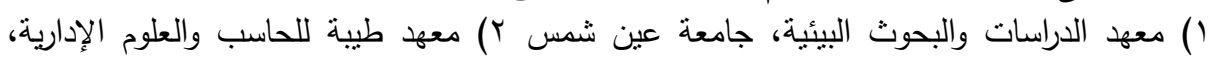
أكاديمية طيبة ب) الجهاز المركزى للتعبئة العامة والإحصاء

\section{المستخليف}

يترتب على الإصـابة بالأمراض التى تتتج عن تلوث المياه العديد من التكاليف، يقع على في

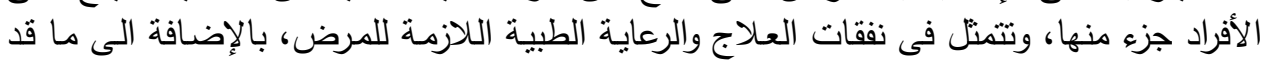
يتسبب عنه خسارة أو فقدان هؤلاء الأفراد من مكاسب قد حال مال المرض دون دون تحققها، وتهدف هذه

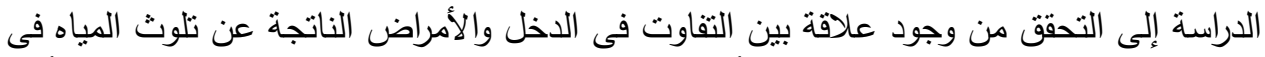

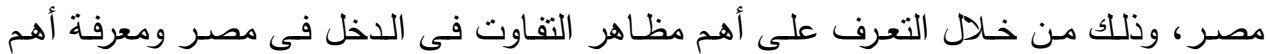

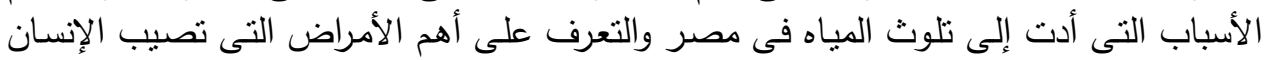

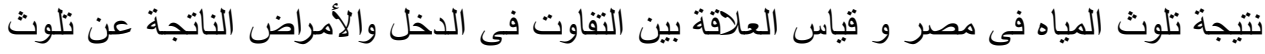

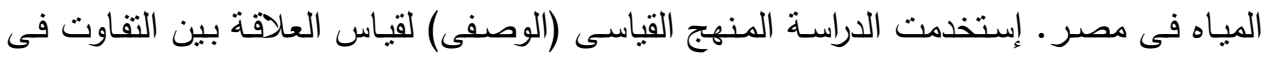
اللدخل والأمـراض الناشـئة عن تلـوث الميـاه بإستخدام طريقة المربعـات الصـغرى العاديـة لبيانـات

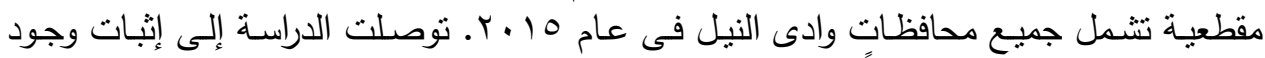

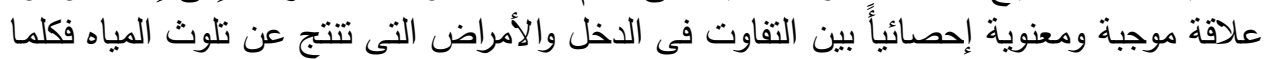
زادت درجة التفاوت فى الدخل زادت أعداد المصابين، وأن العلاقة بين التعليم والأمراض التهن الناشئنة

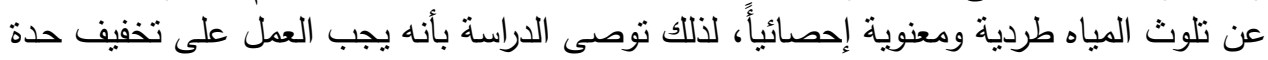

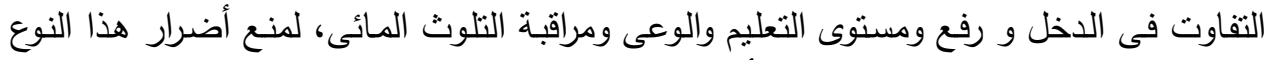

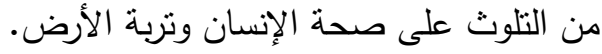

\section{Axas}

تلوث المياه هو أي تغير فيزيائي أو كيميائي في نوعية المياه، بطريق مباشر أو غير

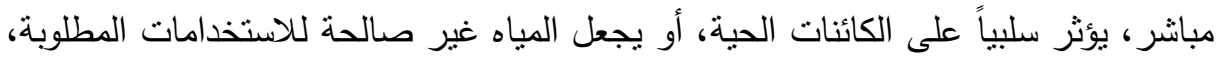

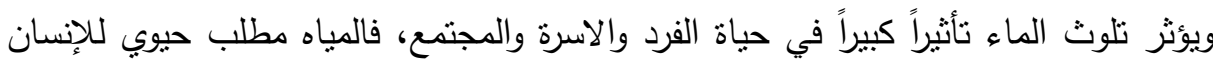

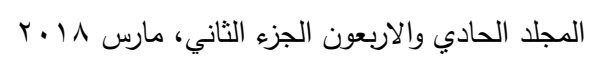


وسائر الكائنات الحية، وقد يكون سبباً رئيسياً في إنهاء الحياة على الأرض إذا كان ملوثاً، فالتلوث الطبيعي للمياه قد يكون نتيجة لتغير خصائصه الطبيعية مثل اللون أو الطعم أو لونياه

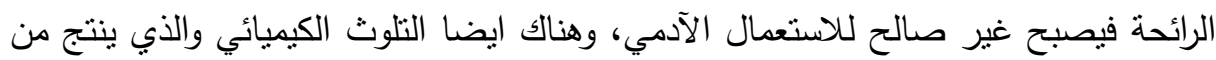

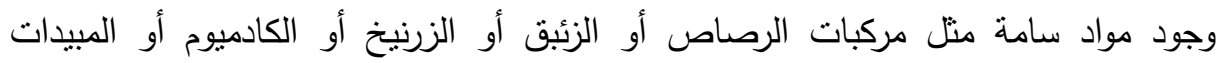

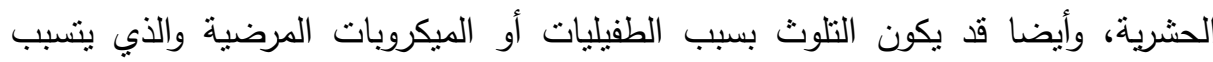
باستعماله في الإصابة بالأمراض المعدية منل البلهارسيا والانكلستوما والاسكارس ( مركز

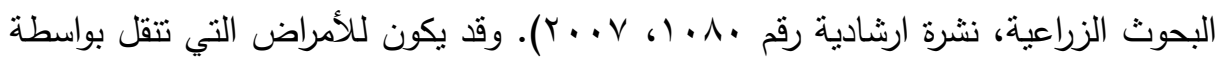
المياه تأثير كبير على الاقتصاد، محليا وكذلك على الصعيد الوطني، ويواجه عادة الأفراد

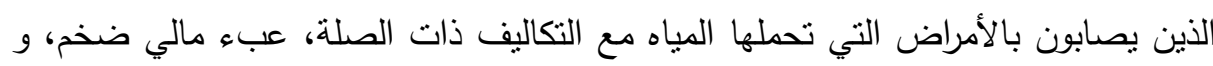

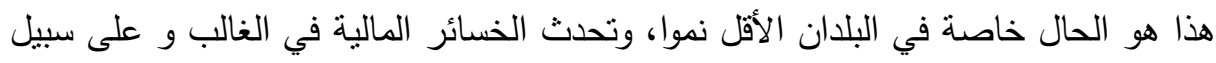
المثال من قبل تكاليف العلاج الطبي والأدوية، وتكاليف النقل والمواد الغذائية الخاصة، وبسبب فقدان الأيدى العاملة، وتضطر العديد من الأسر أن تلجأ إلى بيع أراضيهم لدفع

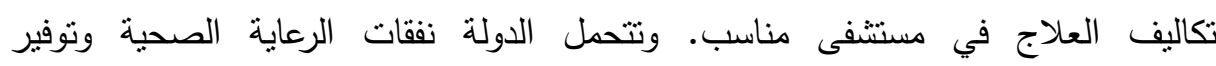
الاستثمارات الضخمة اللازمة لبناء المستشفيات واستيراد الاجهزة والمعدات والادوية، بالاضافة إلى أجور الاطباء والفنيين وهيئات التمريض. ليناء

\section{And Xan}

تأخذ ظاهرة الفقر والبيئة إهتمام كبير على المستوى العالمى حيث إهتم العديد من الباحثين

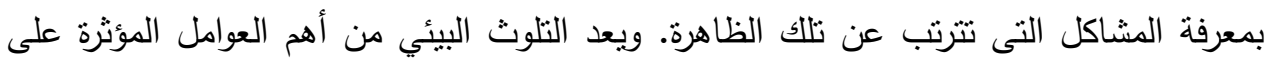

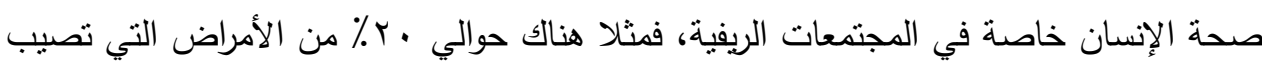

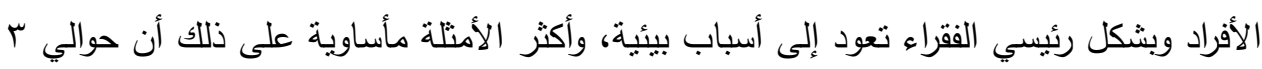

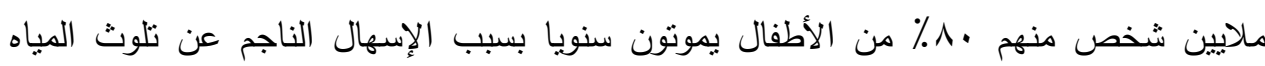

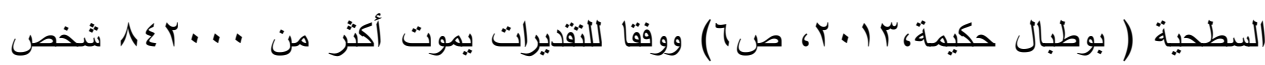
سنويا بسبب الإسهال نتيجة لمياه الثرب غير المأمونة والصرف الصحي وعدم الاعتتاء بنظافة 
الأيدي، والإسهال من الأمراض التي يمكن الوقاية منها إلى حد كبير حيث بمكن تفادي وفاة نحو

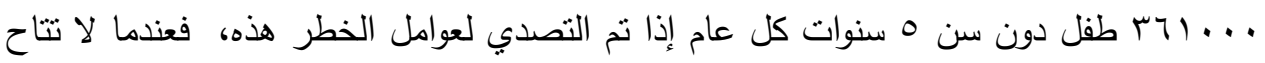

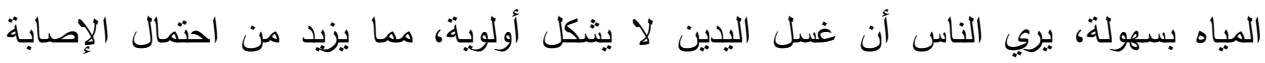

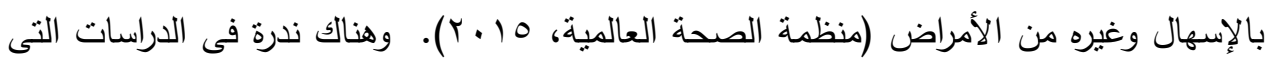

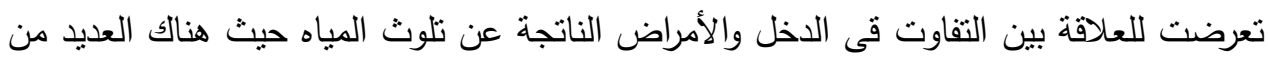

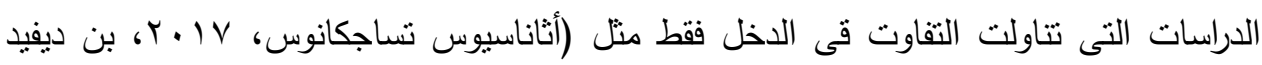

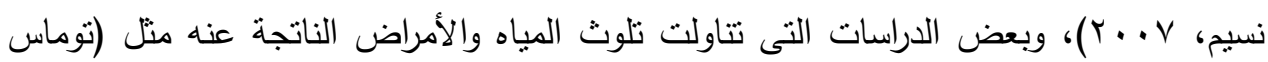

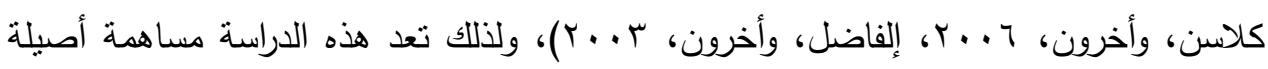

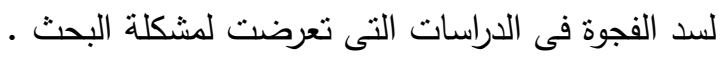
ويتمثل التساؤل الرئيسى للاراسة فى الأتى: هل التفاوت فئى مستوى الاخل احد الاسباب

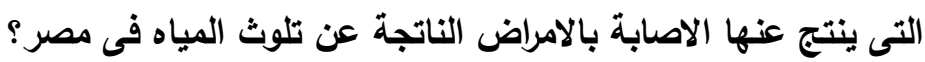

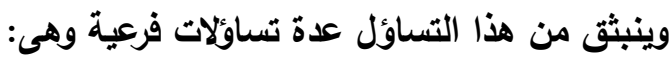

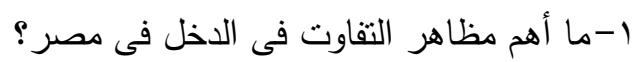

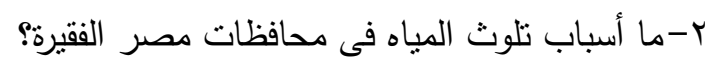

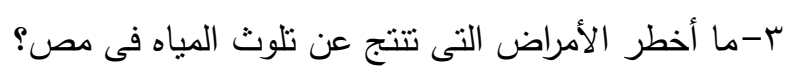
ع- ما مدى وجود علاقة بين التفاوت فى الدخل والامراض الناتجة عن تلوث لثون المياه؟

\section{أمساهيت الهمبه}

تهدف الدراسة إلى إثبات صحة أو خطأ فرضية الدراسة ويتحقق هذا الهرف من خلال

$$
\text { الاهداف الفرعية الاتية: - الاتية }
$$

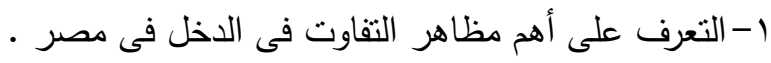

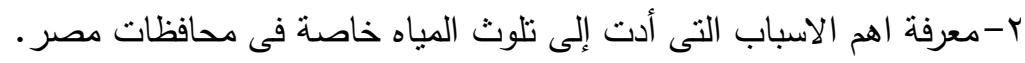

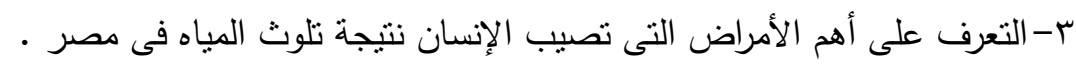

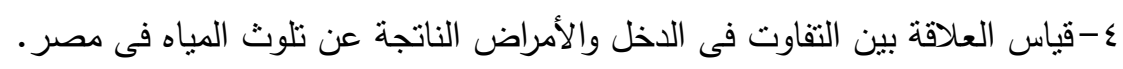

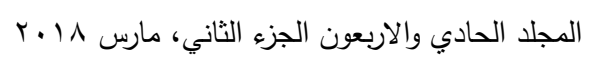


فرضية البحث: وتتمثل فرضية البحث فى: هناك علاقة علاقة احصائية ذات دلالة بين التفاوت فى مستوى الدخل والامراض الناتجة عن تلوث المياه فى محافظات مصر . منهج البحث: من أجل إثبات صحة أو خطأ الفرض إستخدم الباحثة أساليب التحليل

• المنهج الوصفى: حيث قامت الباحثة بتثبع النظريات المتعلقة بتفسير اثز التقاوت فى

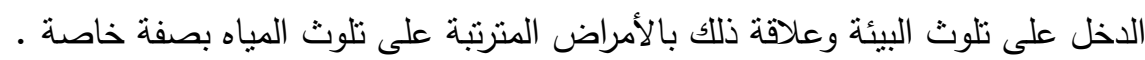
المنهج التحليلى : حيث قامت الباحثة بتجميع البيانات والدراسات عن الظاهرة ثم تفسيرها

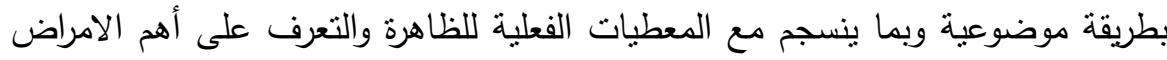
الناشئة عن تلوث المياه وعلاقة ذلك بمستوى الدخل. المنهج القياسى: قامت الباحثة بتصميم نموذج لقياس أثز التفاوت فى الدخل وعلاقته

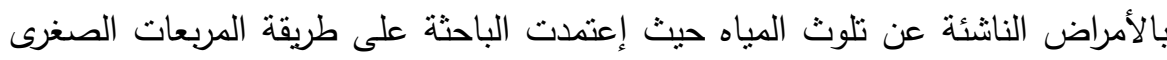
العادية للتعرف على أهم العوامل التى تؤثر على الأمراض الناتجة عن تلوث التهدئ المياه.

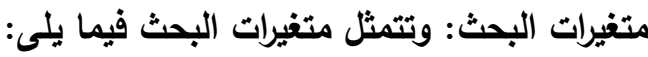

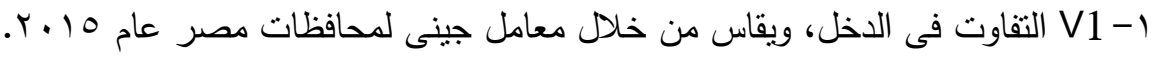

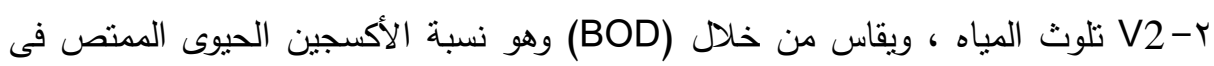

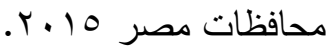
r-V3 الامراض الناتجة عن ثلوث المياه ويقاس من خلال أعداد الإصابة بمرض التهاب الكبدى A على مستوى المحافظات عام 10 . r. ع- التعليم ويقاس من خلال أعداد التلاميذ فى التعليم الأساسى على مستوى المحافظات عام 10. مصادر البيانات: وتتمثل أهم مصادر البيانات فى: التقرير السنوى لإحصاءات البيئة الصادر عن

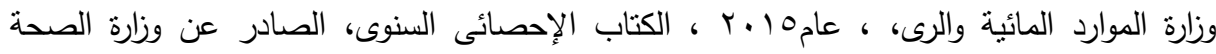

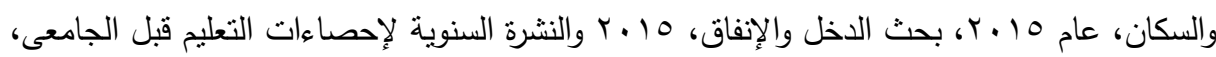

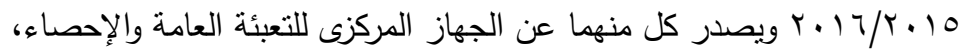




\section{أهمري المهمه}

إن تلوث المياه يشكل خطرا على البيئة، فإن لم يكن نظيفا فسنموت عطشا، والنفايات

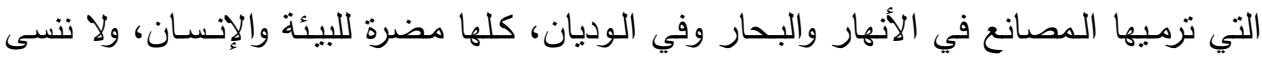
الحيوان وأيضا النبات، كل هذه الكائنات تضر بسبب طرف واحد، و إذا بقينا هكذا فسيدمر العـالم كله وستموت كل الكائنات. وتعد مشكلة تلـوث المياه فى مصر من المشكلات الكبيرة التي بدأت فى الظهور وأخذت فى التزايد، مما استدعى التفكير الجاد فى إيجاد سبل مكافحتها

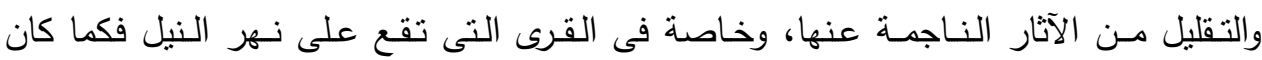
النهر مصدراً لكل متطلبات الناس من المياه فى المقابل كانت تلقى المخلفات والفضلات وحتى

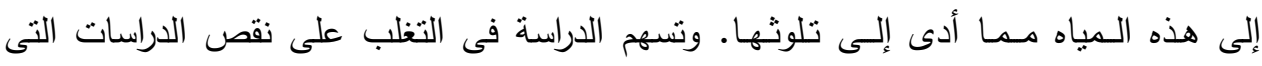
تتاولت هذه المشكلة من خلال محاولة التعرف على قيمة وإتجاه العلاقة بين التفاوت فى الدخل والأمراض الناتجة عن تلوث المياه، ومن أهم الجهات التى تستفيد من هذه الدراسة، (وزارة الصحة

والسكان، وزارة البيئة، وزارة المالية) حدود الدراسة: تشمل الدراسة الدحافظات الواقعة فى وادى النيل وتم إستبعاد المحافظات ورات الحدودية نظراً لعدم نوافر بعض البيانات وتقتصر الدراسة على عام واحد فقط وهو عام

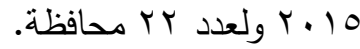

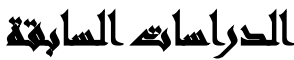

وتتمثل أهم الاراسات السابقة فيما يلى:

1- دراسة محمد عبدالناصر الزرقة بعنوان " تلوث المياه فى محافظتى الثمال والوسطى لهیى

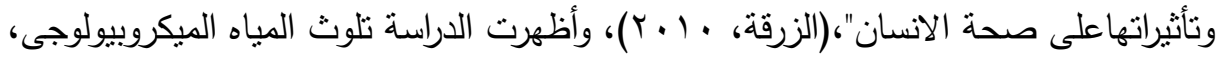

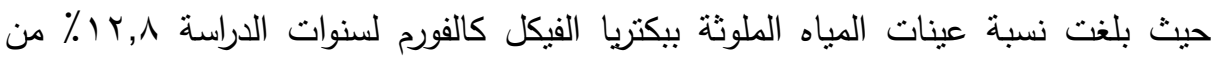

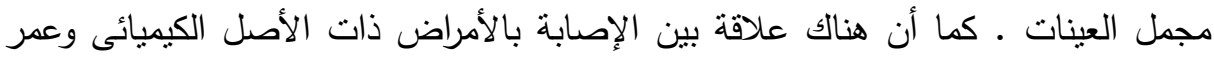

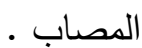


r-دراسة لاثثين، إلخولي، شارابي، الثريف، بعنوان " تقييم المعادن الثقيلة المختارة في بعض

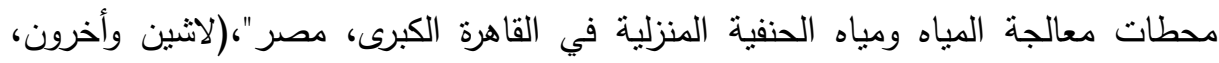

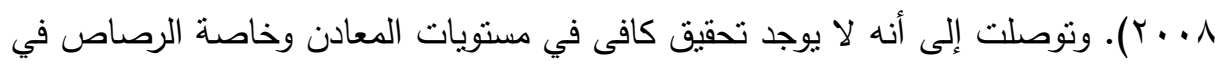

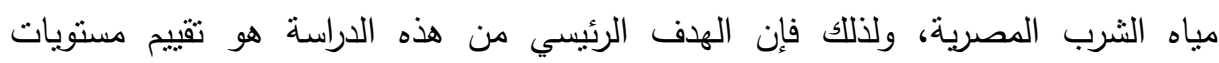
الرصاص في مياه الثرب ومن خلال مقارنة مستويات الرصاص بين كمية معالجة المياه والمياه النهائية يثير إلى أن محطات معالجة المياه لديها كفاءة عالية لإزالة الرصاص. r- دراسة إدوارد، أوبويل ميد أوبويل، بعنوان "الفقر العالمي والجوع والموت والمرضيل، إنهاه (إدوارد

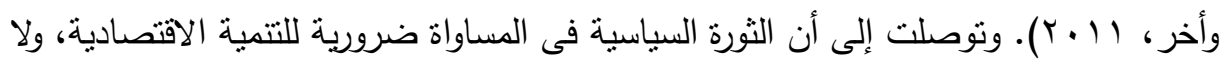

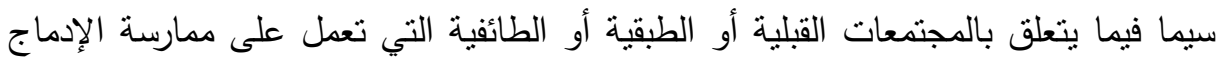
المنهجي للبعض واستبعاد الآخرين ومع ذللك، فإن السعي لتحقيق المساواة يواجه القيود المفروضة على الحرية الفردية اللازمة لإطلاق العنان للطاقات التنافسية. وأن رفاه الإنسان يعتمد بشكل حاسم على العلم والتكنولوجيا بطرق واضحة حتى بالنسبة للمراقب العادي، فعلى لإنى

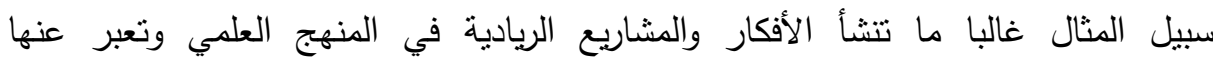
التكنولوجيا في شكل منتجات وخدمات جديدة ومواد جديدة وعمليات إنتاج جديدة.

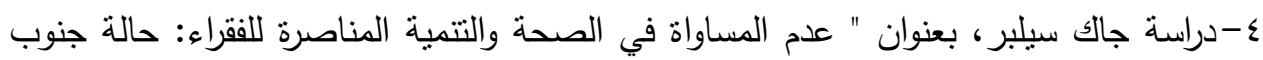
شرق آسيا" (سيلبر ، 10 • ب). وتوصلت إلى إنى أنه بحساب أربعة متغيرات صحية وهى وفيات

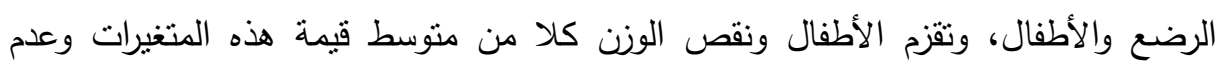

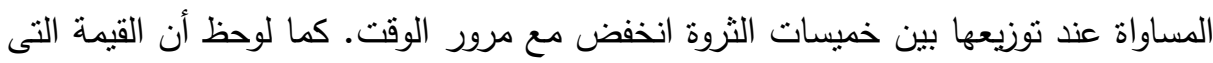
اتخذتها هذه المتغيرات الصحية بين أفقر شريحة للثروة كانت أساسا نتيجة لذلك.

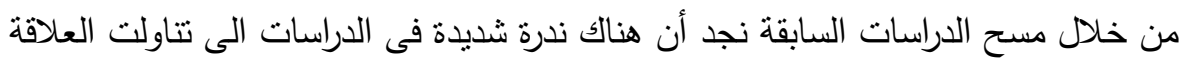

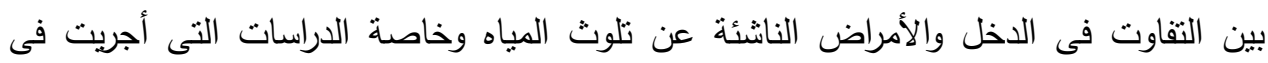
مصر حيث تتاولت هذه الدراسات أحد جوانب الدراسة الحالية دون الأخرى ومن ثم تعد دراستتا

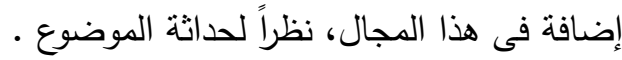




\section{العلاقة بين التفاوت فى الاخل والأمراض الناشئة عن تلوث المياه:}

يفسر بعض علماء الإقتصاد التباين فى مستويات الدخول بالتفاوت فى الفرص المتاحة، فالبيئة التى يعيش فيها الأفراد تلعب دور هام فى تحديد الدخل الحالى والمستقبلى لهم، حيث تقدم الهياكل الإقتصادية والإجتماعية نسهيلات تهيئ الفرصة للأفراد للحصول على الوظائف والأعمال ذات الدخل العالى. وقد تختلف هذه الفرص بإختلاف المناطق الجغرافية فأبناء المدن فرصهم الجناء أعلى من ابناء الريف فى الحصول على الوظائف، وقد تختلف الفرص أيضاً بسبب الهياكل المؤسسية كالقوانين أو العقائد أو اللون أو الجنس. و قد تختلف أيضا بسبب المخاطرة حيث اليثاء يخاطر البعض بممارسة الأعمال الخطيرة ويغامرون بينما يحجم غيرهم عن ذلك بسبب المخاطرة

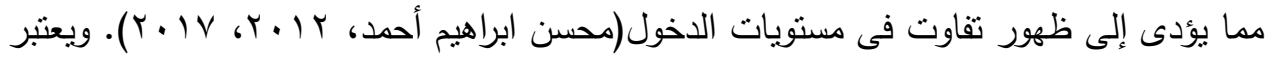

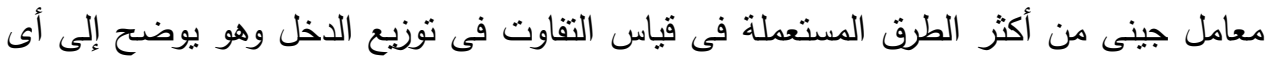

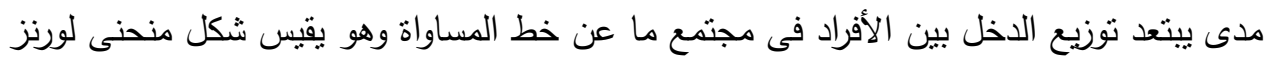

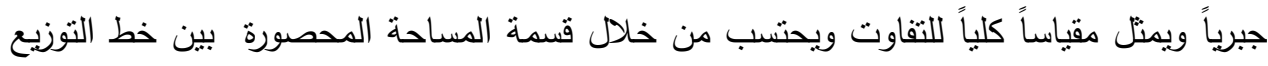
المتساوى ومنحنى لورنز على المساحة الكلية للمتلث الذى يقع فيه المنحنى او المساحة الكلية تحت خط التوزيع المنساوى ـ تقع قيمة معامل جينى بين حدين، يمثل حده الأدنى ويساوى صفر ،

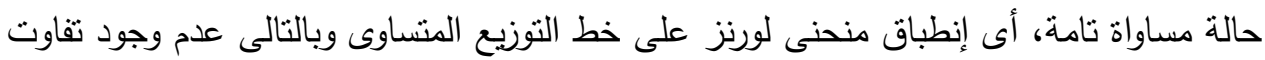

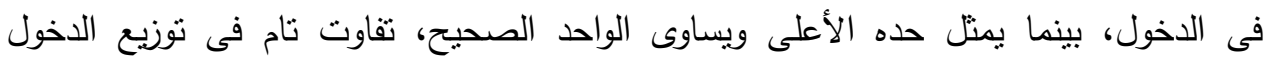

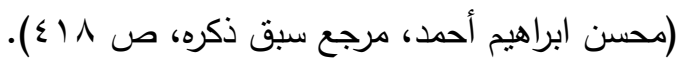

وترتبط المياه الملوثة وتردي خدمات الصحة بانتقال الأمراض مثل الكوليرا والإسهال

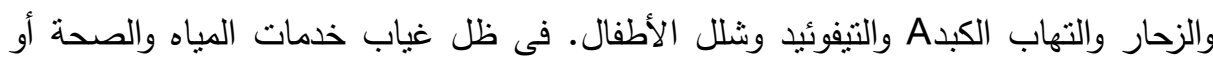
عدم كفايتها أو سوء إدارتها، يمكن أن يتعرض الأفراد إلى مخاطر صحية بمكن تلافيها

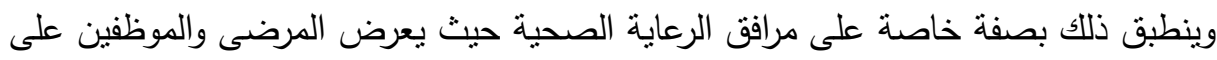
حد سواء، للمزيد من مخاطر العدوى والمرض في غياب خدمات المياه والصحة والنظافة. وفي لرعابه

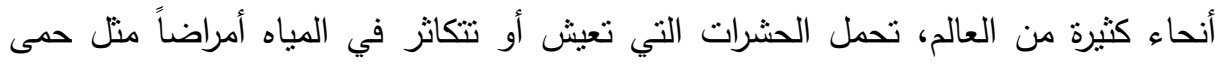

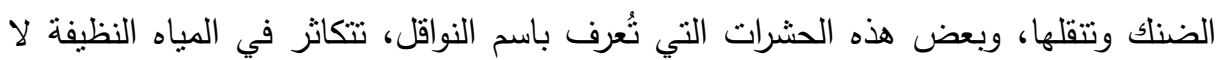

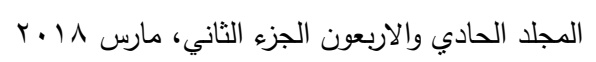


في المياه القذرة، ويمكنها أن تستخدم حاويات مياه الثرب المنزلية كأماكن لتكاثزها، والتنخل

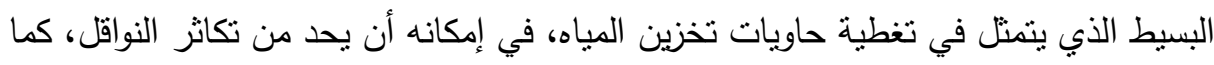

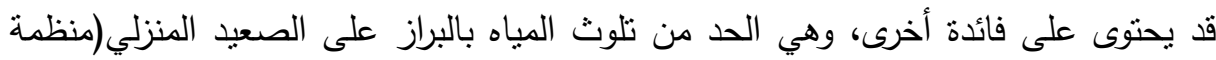

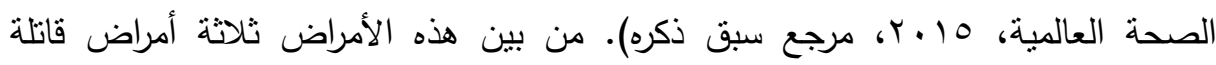

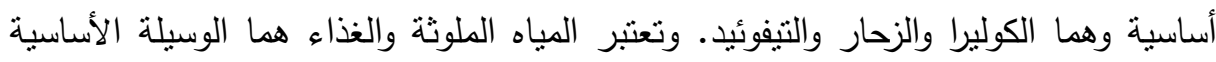
التي تتنتر بها هذه الأمراض. وهناك ما يقدر بنحو بليوني طفل بصابون بهذه الأمراض كل ولئر

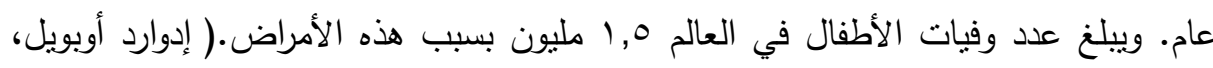

\section{المئائج والميوكياني}

أولاً: النتائج: ويمكن توضيح العلاقة بين التفاوت فى الدخل والأمراض الناشئة عن تلوث المياه من خلال تتبع الجداول التالية، حيث يبين الجدول رقم (1) درجة التفاوت فى الدخل ونسبة الفقراء

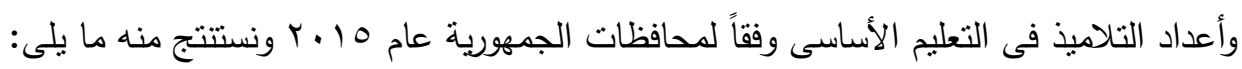

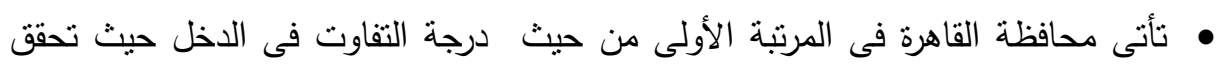

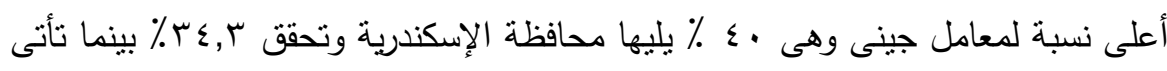

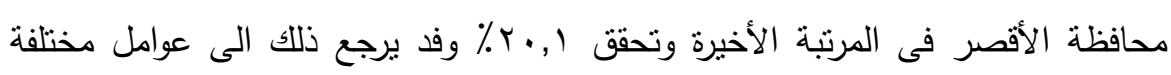

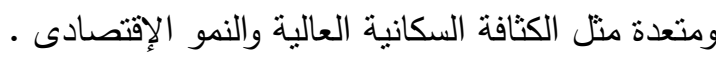

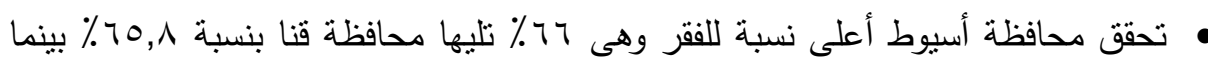

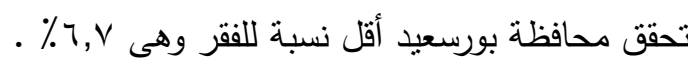

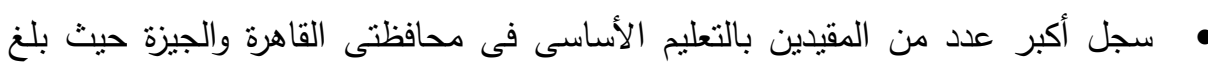

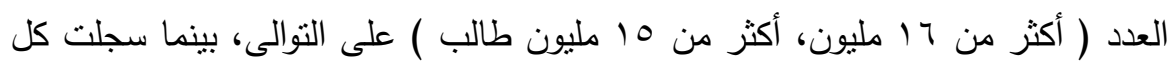

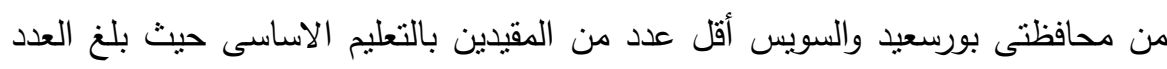

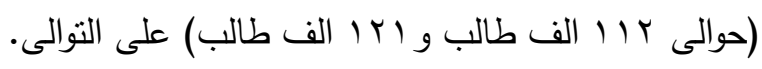


• سجلت القياسات نواجد الأكسجين الحيوى الممتص في الحدود المسموح بها في أغلب

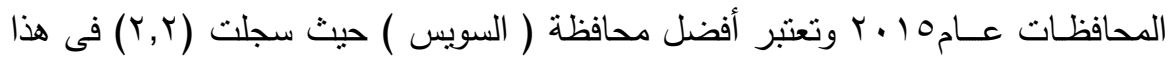

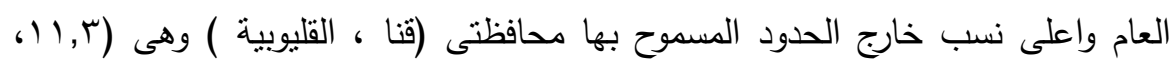
( ) $11, r$ جدول رقم( ) : درجة التفاوت فى الدخل ونسبة الفقراء وأعداد التلاميذ فى التعليم الأساسى وفقاً

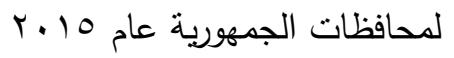

\begin{tabular}{|c|c|c|c|c|}
\hline الملوث المائى (BOD) & أعداد التلاميذ فى التعليم & الفقراء & معامل & المحافظات \\
\hline 1,0 & $17 . V T M T$ & $1 V, 0$ & $\varepsilon$. & القاهرة \\
\hline$r, \wedge$ & ヘฯฯ. ธฯ & 11,7 & $\Gamma \varepsilon, \Gamma$ & الإسكندرية \\
\hline$r, \varepsilon$ & MTYYA & $7, V$ & $r\urcorner, V$ & بورسعيد \\
\hline$r, Y$ & ITIMTI & $\mid V, 1$ & $r q, r$ & السويس \\
\hline$\varepsilon, 9$ & $r V \leq \ldots r$ & 11 & ro, 9 & دمياط \\
\hline 7,9 & 11.0911 & 10,1 & $Y 0,1$ & الدقهلية \\
\hline $7, r$ & IYTVYAY & $1 \varepsilon, 1$ & $r r, 0$ & الشرقية \\
\hline $11, r$ & 9911.7 & $1 T, 1$ & $r 0, Y$ & القليوبية \\
\hline $7, Y$ & 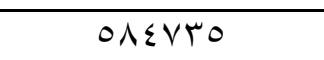 & 19,8 & $r r$ & كفرالثيخ \\
\hline$\varepsilon, \varepsilon$ & 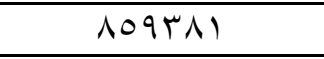 & 17,0 & $r 0, V$ & الغربية \\
\hline$\varepsilon, 0$ & $V 779.0$ & 17 & $Y 0, V$ & المنوفية \\
\hline 0,7 & $111 \cdot \leq r \wedge$ & $r T, V$ & YI,V & البحيرة \\
\hline$r, r$ & rrqr人r & $r \varepsilon, 1$ & YY, & الإسماعيلية \\
\hline$\Lambda, 0$ & lo9VArr & $r \wedge, 7$ & $r q, r$ & الجيزة \\
\hline$\varepsilon, \cdot$ & $0 \leqslant Y Y I 1$ & $\varepsilon r, 1$ & $r \leq$ & بنى سويف \\
\hline$\varepsilon, \cdot$ & 0人r770 & $r 0, v$ & $r \leqslant, 0$ & الفيوم \\
\hline $0, \cdot$ & 9901117 & $07, \mathrm{~V}$ & $r V, 1$ & المنيا \\
\hline$r, \Lambda$ & $\Lambda 17 \varepsilon \cdot 7$ & 77 & $Y V, Y$ & أسيوط \\
\hline$r, v$ & $1979 . r$ & 70,1 & Yo,V & سوهاج \\
\hline $11, r$ & OVTrVq & $O V, \Lambda$ & $Y 0,1$ & قنا \\
\hline $0, r$ & $r \cdot r \varepsilon . q$ & $\{\wedge, 7$ & $Y 0,1$ & أسوان \\
\hline $0, r$ & TOYNT & $\sum 1, r$ & $r \cdot, 1$ & الأقصر \\
\hline
\end{tabular}


المصدر: الجهاز المركزى للتعبئة العامة والإحصاء، (بحث الدخل والإنفاق،10 • ب/1 • ب، النشرة

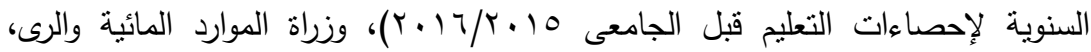

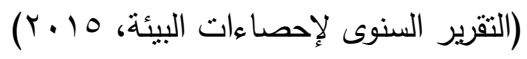

ويبين جدول رقم (r) عدد حالات الاصابات والوفيات للأمراض المعدية المبلغ عنها على مستوى المحافظات خلال عام 0 ـ ب ونستتج منه ما يلى: أن محافظة الثرقية هى الأعلى من حيث عدد حالات الإصابة بمرض التيفويد حيث

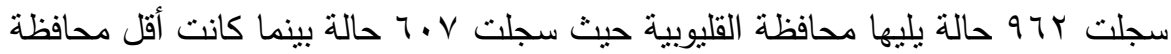
من الاصابة بمرض التنفويد كانت محافظة بورسعيد حيث سجلت 0 حالات أصابة فقط يليها محافظتى القاهرة وجنوب سيناء بعدد V حالات أصابة فقط. ولم يتم تسجيل أى حالة

$$
\text { وفاة بسبب هذا المرض. }
$$

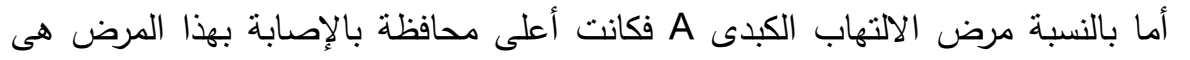

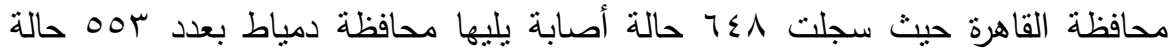
إصابة وكانت أقل محافظة من حيث عدد حالات الأصابة بهذا المرض هى محافظة مطروح حيث سجلت حالة وحيدة مصابة بهذا المرض، يليها محافظة المنيا حيث سجلت

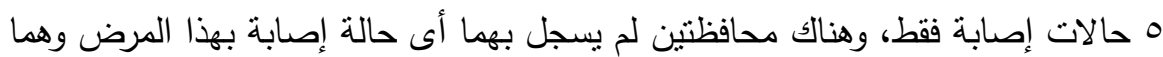
محافظتى الوادى الجديد وشمال سيناء ـ كما يوجد ؛ حالات وفاة بسبب مرض الالتهاب الكبدى A هذه الحالات سجلت فى محافظات الدقهلية وسجلت حالتين وفاة و الإسكندرية وسجلت حالة وفاة واحدة والجيزة أيضاً سجلت حالة وفاة واحدة. بالنسبة لمرض النزلة المعوية (الإسهال) فكانت أعلى محافظة من حيث عدد حالات الإصابة بهذا المرض هى محافظة القليوبية حيث سجلت rV rV حالة إصابة يليها

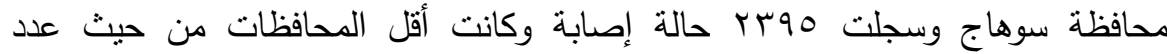
حالات الإصابة المسجلة لهذا المرض هما محافظتى السويس والوادى الجديد حيث سجل بكل منهما حالة إصابة واحدة ـ ولم يتم تسجيل أى حالة وفاة بسبب هذا المرض. 
مصطفى حسن رجب وآخرون

جدول رقم(r): عدد حالات الاصابات والوفيات للأمراض المعدية المبلغ عنها على مستوى

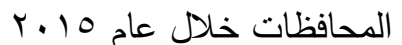

\begin{tabular}{|c|c|c|c|c|c|c|}
\hline \multicolumn{2}{|c|}{ النزلة المعوية_(الاسـهال) } & \multicolumn{2}{|c|}{ التهاب كبدى A } & \multicolumn{2}{|c|}{ التيفويد } & \\
\hline الوفيات & الاصاباث & الوفيات & الاصابات & الوفيات & الاصابات & \\
\hline . & 199 & . & $7 \leqslant \wedge$ & . & $\mathrm{V}$ & القاهرة \\
\hline . & 177 & 1 & $\sum Y I$ & $\cdot$ & $r \wedge$ & الإسكندرية \\
\hline$\cdot$ & $1 \wedge V$ & $\cdot$ & 17 & $\cdot$ & 0 & بور سعيد \\
\hline - & 1 & - & $1 \wedge 1$ & - & ro & السويس \\
\hline . & ry & . & $V \varepsilon$ & . & $11 \% 0$ & الإسماعيلية \\
\hline . & TME & $\cdot$ & $00 \%$ & $\cdot$ & MV & دمياط \\
\hline . & 11 & $r$ & 109 & . & 91 & الدقهنية \\
\hline$\cdot$ & $09 \varepsilon$ & $\cdot$ & YI. & $\cdot$ & $97 r$ & الشرقية \\
\hline$\cdot$ & $r V \leqslant r$ & $\cdot$ & 77 & . & $7 \cdot V$ & القليوينية \\
\hline$\cdot$ & 1017 & . & 119 & . & 01 & كفز الشيخ \\
\hline$\cdot$ & rT & $\cdot$ & YIV & $\cdot$ & 11. & الغزيبة \\
\hline . & TrV & $\cdot$ & OY & . & YOS & المنوفية \\
\hline$\cdot$ & $r$ & . & 107 & . & $0 \leqslant 1$ & البحيرة \\
\hline$\cdot$ & $1 \varepsilon$. & 1 & $\varepsilon \varepsilon Y$ & $\cdot$ & 18 & الجيزة \\
\hline . & MIN & . & $\varepsilon r$ & . & 115 & بنى سويف \\
\hline$\cdot$ & $V \leqslant 1$ & $\cdot$ & rr & $\cdot$ & $r \wedge$ & الفيـوم \\
\hline . & $9 \wedge r$ & . & 0 & . & VVA & المنيـا \\
\hline$\cdot$ & 1870 & $\cdot$ & 107 & $\cdot$ & rE. & أسيوط \\
\hline - & rrqo & - & IV & . & 111 & سوهاج \\
\hline . & $\varepsilon$ & . & 99 & . & $1 \leqslant Y$ & L \\
\hline . & $1 \cdot \leq \varepsilon$ & . & $V_{1}$ & . & 11 & أسوان \\
\hline$\cdot$ & $V \varepsilon$ & $\cdot$ & 181 & $\cdot$ & rTr & مدينة الأقصر \\
\hline
\end{tabular}

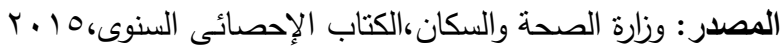
قياس العلاقة بين التفاوت فى الاخل والأمراض الناشئة عن تلوث المياه: ويمكن قياس العلاقة

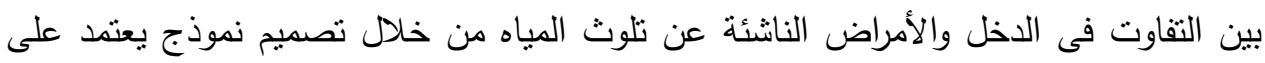

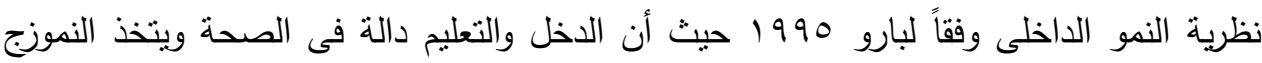

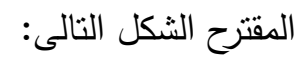

$$
\mathbf{H}=\boldsymbol{\beta}_{\mathbf{0}}+\boldsymbol{\beta}_{\mathbf{1}} \mathbf{I}+\boldsymbol{\beta}_{\mathbf{2}} \mathbf{E}+\boldsymbol{\beta}_{3} \mathbf{P}+\epsilon_{i}
$$




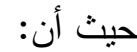

الأمراض ( أعداد الإصابة بمرض الإلتهاب الكبد A لمحافظات جمهورية مصر العربية

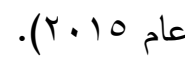

.

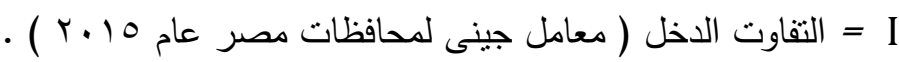

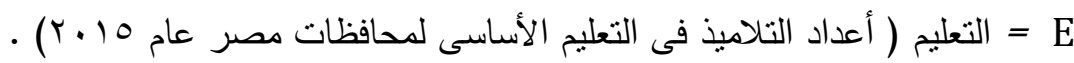

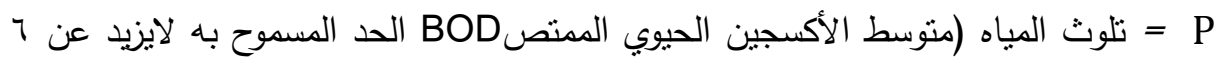

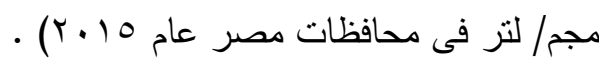
=

تم إختيار مرض الالتهاب الكبدى A كوكيل عن ( PROXY) الأمراض الناشئة عن

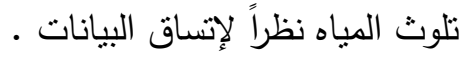

وإستخدمت الباحثة المنهج القياسى بطريقة المربعات الصغرى العادية لبيانات مقطعية

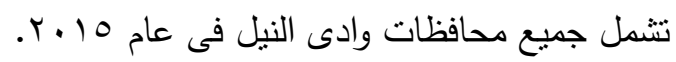

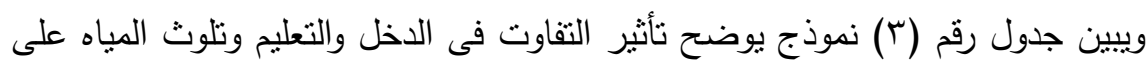

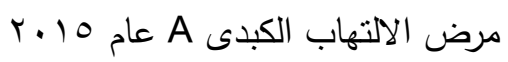

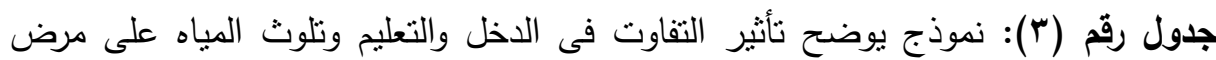

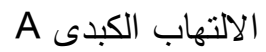

\begin{tabular}{|c|c|c|c|c|}
\hline Prob. & z-Statistic & Std. Error & Coefficient & Variable \\
\hline \hline 0.0001 & -3.904095 & 158.2965 & -618.0046 & C \\
\hline 0.0000 & 4.149431 & 6.010000 & 24.93808 & I \\
\hline 0.0162 & 2.405068 & $6.57 \mathrm{E}-05$ & 0.000158 & $\mathbf{E}$ \\
\hline 0.9472 & 0.066197 & 10.47111 & 0.693153 & P \\
\hline
\end{tabular}

المصدر: نم بمعرفة الباحثة 
يتبين من جدول رقم (r) ما يلى

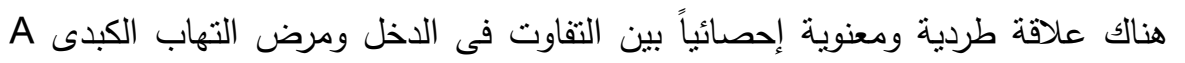

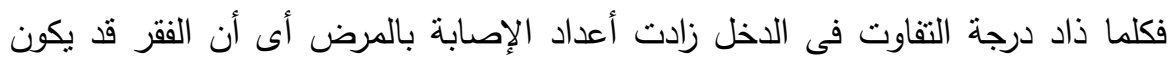
سبباً أساسياً فى الإصابة بهذا المرض وقد يرجع ذلك إلى عدة أسباب منل عدم إمكانية الحصول على مياه صالحة للإستعمال وعدم توافر صرف صحسى لإنى مناسب. توجد علاقة طردية ومعنوية إحصائياً بين التعليم ومرض الإلتهاب الكبدى A ألى ألى أنه كلما

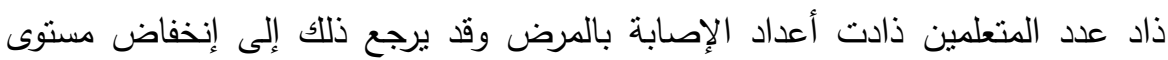
التعليم ونقص الوعى الصحى. • العلاقة بين نلوث المياه (BOD) ومرض الإلتهاب الكبدى A كانت طردية ولكن غير معنوية إحصائياً، وتعد هذه النتيجة غير منوقعة، ولكن قد يرجع ذلك إلى عدم كفاية الإنهاب البيانات.

ثانياً: التوصيات: بناءاً على الإستتاجات التى تم التوصل اليها، نقترح ما يأتى:

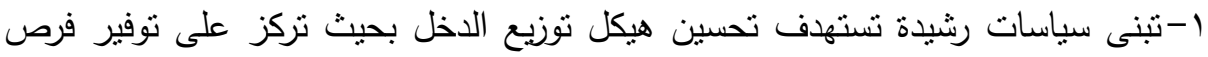

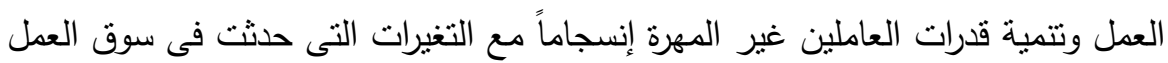
نتيجة ثورة التكنولوجية والمعلومات والإتصالات، وفى هذا المجال فإن ذيادة الإستثمارات وإعادة تخصيصها بين الإستخدامات المختلفة تعد الأداة الرئيسية لرفع وتحسين معدل ولهد ولهال

$$
\text { النمو الإقتصادى وتخفيف حدة التقاوت. }
$$

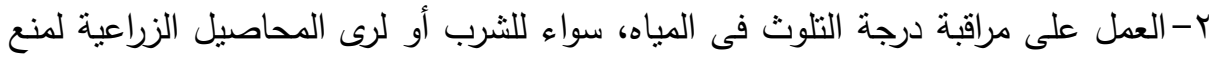

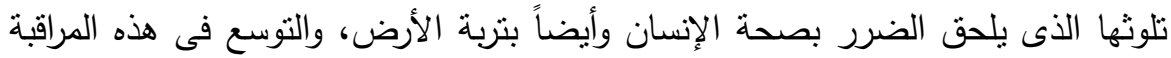

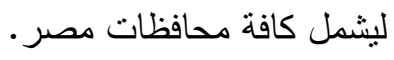

r-من أجل ذيادة متوسط دخل الفرد وتفليل درة التفاوت، لابد من رفع كفأة العاملين وزيادة إنتاجيتهم، ومساعدة خريجى الجامعات والمعاهد والفئات العاطلة عن العمل والفئات ذات الدخل المنخفض فى إيجاد سبل التمويل اللازم لإقامة المشاريع الصغيرة التى تعند على عنصر العمل والتكلفة المنخفضة، من خلال منح قروض قصيرة الأجل.

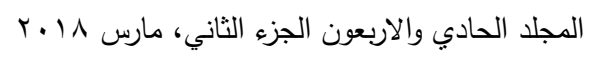


ع- العمل على رفع مستوى جودة التعليم وذيادة الإهتمام بنشر الوعى الصحى بين أفراد المجتمع

\section{zall}

بوطبال حكيمة، رباحى فضيلة، إنكالية الفقر والبيئة، جامعة سعد دحلب البليدة، ص77. الجهاز المركزى للتعبئة العامة والإحصاء، النشرة السنوية لإحصاءات التعليم قبل

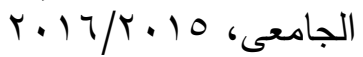

الجهاز المركزى للتعبئة العامة والإحصاء، بحث الاخل والإنفاق، 10 ب r

محسن ابراهيم أحمد، "قياس وتحليل التفاوت فى الدخل فى محافظة السليمانية"، مجلة ولاهية

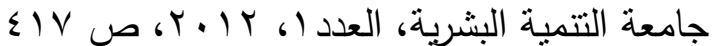

محسن ابراهيم أحمد، مرجع سبق ذكره، ص

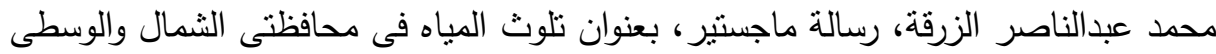

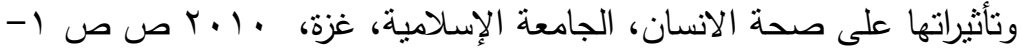
171

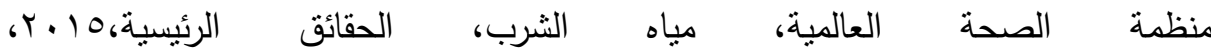
int/mediacentre/factsheets/fs391/ar

منظمة الصحة العالمية، ، 10 • ب، مرجع سبق ذكره

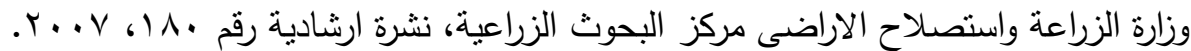
وزارة الصحة والسكان، الكتاب الإحصائى السنوى، 10 ـ ب.

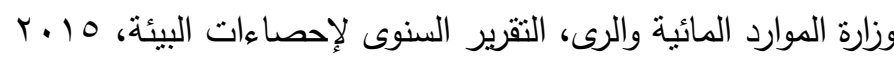

Athanasios Tsagkanos, (2017) "Stock market development and income inequality", Journal of Economic Studies, Vol. 44 Issue: 1, pp.87-98.

Ben David Nissim, (2007) "Economic growth and its effect on income distribution", Journal of EconomicStudies, Vol. 34 Issue: 1, pp.42-58.

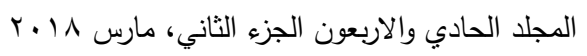


Edward O'Boyle, Meade O'Boyle, (2011) "Global poverty, hunger, death, and disease", International Journal of Social Economics, Vol. 39 Issue: 1/2, pp.4-17.Jacques Silber, (2015) ", "On inequality in health and pro-poor development: the case of Asia Southeast. Journal ojkkjjkjkf Economic Studies, Vol. 42 Issue: 1, pp.34-53.

Edward O'Boyle, Meade O'Boyle, Previous reference, ${ }^{2011, p p .4-17 .}$

M. El-Fadel, R. Maroun, L. Semerjian, H. Harajli, (2003) "A healthbased socio-economic assessment of drinking water quality: the case of Lebanon", Management of Environmental Quality: An International Journal, Vol. 14 Issue: 3, pp. 533368.

M.R. Lasheen, G. ElKholy, C.M. Sharaby, I.Y. Elsherif, S.T. ElWakeel, (2008) "Assessment of selected heavy metals in some water treatment plants and household tap water in Greater Cairo, Egypt", Management of Environmental Quality: An International Journal, Vol. 19 Issue: 3, pp.367376.

Thomas Clasen, Lucy Smith, Jeff Albert, Andrew Bastable, Jean Francois Fesselet, (2006) "The drinking water response to the Indian Ocean tsunami, including the role of household water treatment", Disaster Prevention and Management: An International Journal, Vol. 15 Issue: 1, pp.190-20

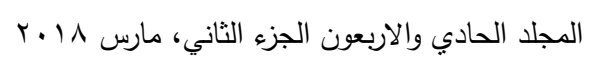


مجلة العلوم البيئية

معهد الدراسات والبحوث البيئية - جامعة عين شمس لمس لمس

\title{
THE IMPACT OF INCOME INEQUALITY ON
}

DISEASES CAUSED BY WATER POLLUTION IN

EGYPT

\author{
Ragab, M. H. ${ }^{(1)}$; Rezkallah, W. W. A. ${ }^{(2)}$ \\ and Abdul Jawad, Rabab, K. $^{(3)}$
}

1) Institute of Environmental Studies and Research, Ain Shams University 2) Thebes Higher Institute for Computer and Management Sciences, Thebes Academy 3) the Central Agency for Public Mobilization and Statistics

\begin{abstract}
Diseases caused by water pollution lead to many costs, The Individuals incur part of it, Like the costs of treatment and medical care for the disease, in addition to the loss of benefits Which causes the disease In failing to achieve them, The aim of this study is to investigate the relationship between income inequality and diseases Caused by water pollution in Egypt, By know the most important aspects of income inequality in Egypt, And know the most important reasons that led to water pollution in Egypt, And know the most important diseases that affect humans as a caused by water pollution in Egypt, And measuring the relationship between income inequality and diseases caused by water pollution in Egypt. To measure the relationship between income inequality and diseases resulting from water pollution the standard approach was used using the method of the micro squares normal for the cross Sectional data covering all governorates of nile Valley in 2015. The study found that there is a positive Statistically significant relationship between income inequality and diseases caused by water pollution, And that the relationship between education and diseases caused by water pollution is both positive and Statistically significant. So must the work on Relieve$$
\text { المجلد الحادي والاربعون الجزء الثاني، مارس 11 بـ }
$$ 
مصطفى حسن رجب وآخرون

intensity income inequality, And upgrading the level of education And water pollution control,for prevent the damage of this type of pollution on human health and soil. 